\title{
Cloud based IoT Applications for Industrial and Home Automation
}

\author{
Aravindh Kolanchinathan, Arockia Selvakumar A
}

\begin{abstract}
IoT for Industrial and Home Automation is emerging with a big bang, has huge potential for every field to be used. When there is a need for efficient means to seek IoT interface, a cloud server is what strikes in every design and applications. There are numerous aspects in building a real-time IoT interface, but IoT through cloud can be a source of multiple gains in contrast to its peers such as edge computing [1]. Industrial and Home Automation involve an excellent delivery protocol for an error-free effective transmission in the internet. MQTT protocol is a better option to facilitate the requirements of IoT through its smooth implementation, Quality of Service and data delivery. In today's world, IoT cloud providers compete to provide reasonable and precise IoT based utilities. Despite extensive engagement of these IoT clouds, we have not initiated standard regularization or few comparative analytical investigations across the research databases. An IoT application calls for diverse resources of a particular cloud and hence it calls for a survey on IoT cloud concerning Latency, interval for subsequent update, user-friendliness, IFTTT compatibility, data handling, processing data, storage limits, servers used and security. An analyses of five of the most eminent clouds (Adafruit IO, Amazon Web Service (AWS), Blynk, Thingspeak and Ubidots) based on the above-described specifications are the factors of motivation for this paper and hence matches the best cloud suited to serve specific purpose and applications.
\end{abstract}

Keywords : Internet of Things (IoT), Message Queuing Telemetry Transport (MQTT), IFTTT (If This Then That).

\section{INTRODUCTION}

T The Internet of Things, or "IoT" [2] [17] [18] in brief, is about enhancing the intensity of the internet beyond computers and smartphones to a whole new dimension of alternative devices, processes and ecosystems. These "hooked up" objects build information and delivers materials back to the system. Home and Industrial automation seeks for excellent quality IoT connection because of its ability to perform tasks with embedded technologies to sense, communicate, interact, and collaborate with things, creating a network of physical objects which fits perfect for the requirements. The applications can be broadly classified as home automation and industrial automation and both has its own requirements, technology, novelty, designs and complexity. In today's world most of the IoT applications are

Revised Manuscript Received on December 16, 2019.

* Correspondence Author

Dr. A. Arockia Selvakumar*, Senior Associate Professor, Design and Automation Research group, School of Mechanical and Building Sciences, Vellore Institute of Technology, Chennai, Tamil Nadu, India.

Email: arockiaselvakumar@yahoo.co.in

Arockia Selvakumar A, Senior Associate Professor, Design and Automation Research group, School of Mechanical and Building Sciences, Vellore Institute of Technology, Chennai, Tamil Nadu, India. implemented in various cloud platforms for its advantage. We may interpret cloud with MQTT [16] [19] as a system that revolves around the "Cloud Computing" base to achieve bulk handling ability \& data to hold its activity that measures all computation, sensing and data merged into a simple independent IoT cloud server. Cloud is crucial in aggregating messages, keeping records and to retrieve statistical insights from those data. It provides high scalability by passing data to the cloud server from all the sensors \& controls present in the equipment that are processed well in aggregate. Hence cloud has had vital conclusions for IoT based applications. There goes many clouds in the internet that are earnestly seeking active strategies and clients to establish their dominance in IoT. Many of these clouds certainly are much fixed to prove their uniqueness and features but less concerned about the standard and the quality of service they provide. In other words, many clouds insist the users how to operate and control their cloud rather than allowing the users to handle the cloud's resources and capabilities. The clouds vary enormously since they have open norms, standards and regulations that are governed by their administrators. Some clouds offer free services, some offer subscription based services and some use for their own applications and it is very crucial to know all the features, requirements, resources and characteristics of a particular cloud before one utilizes a service offered by various cloud present across the internet for his own IoT application.

An IoT application requests for diverse resources in a specific cloud signaling the need for a survey on IoT cloud concerning latency, interval for subsequent update, user friendliness, IFTTT compatibility, data handling, processing data, storage limits, servers used and security. These characteristics are enough to study and evaluate and understand services, functions, support and resources offered by a particular cloud. Hence a comparison is presented on the current versions of the clouds (January, 2020) and are contrasted to determine their features, capabilities and suggestions. This paper surveys various aspects produced in five of the most eminent clouds (Adafruit IO, AWS, Blynk, Thingspeak and Ubidots) based on above described specifications. The user not necessarily has to consider only these five clouds or the features mentioned, but can go for his own choice of cloud service and can contrast them with his own set of evaluative the cloud parameters. The ultimate aim is to contribute precise intelligence about the existing IoT cloud service providers, their pros and cons so that the user can adopt the cloud based on his obligations that leads to standardizing norms and quality of service in the near future [20] [21].

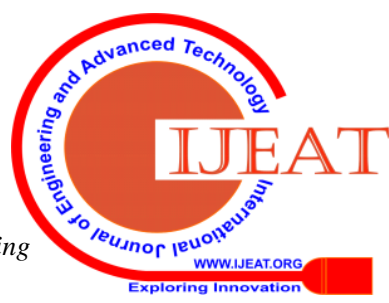




\section{HYPOTHESIS AND EXPLORATION}

The modern day IoT device (ESP8266 NodeMCU) is provided with Wi-Fi Network of $2.4 \mathrm{GHz}$ from a $4 \mathrm{G}$ LTE cellular network of $2 \mathrm{Mbps}$ internet link. Processing time taken by the hardware device and network is not accounted since it is calculated to be in microseconds and hence it is of least significance. The steps were drawn out at unconventional periods of time to have consistent and accurate results. Elements such as location, network linkage, hardware equipment and processors are kept same to establish that the only "variable affected is the server and related parameters". The data may vary when the mentioned parameters are altered. When a cloud/hardware equipment desires to transfer the data constantly, there holds two delays called the cloud latency and update delay. The cloud latency is induced by the travel route of data and the time taken by the cloud to execute the requested action. When the equipment ought to update the cloud channel, the data has to reach a defined location (feed location in the user's account) which incurs path delay. The server takes turns to determine the user's account, then decrypt the data and then to revise the corresponding feed that costs processing delay. The arithmetic summation of these two delays brings out the first delay called the cloud latency. The second delay is observed when the data is updated at periodic intervals by the IoT device to a distinct feed in the cloud. The update delay is encountered by the IoT device since the service providers makes sure their servers are not overloaded with an uninterrupted burst of data from various devices. Hence every server is predetermined with an upload delay of specific intervals based on "client handling and administration capabilities".

Throughput is an essential factor that should be considered for an effective IoT cloud. If the packets are lost, the desired task may not be completed which may lead to failure of the entire IoT system. Hence it is a must for all the clouds to have its throughput of $100 \%$ for its effective utilization. Update delay is also one of the important parameter that decides the speed of the IoT cloud. Uploading many of data in fixed interval implies the cloud works at high speed and less data implies low speed. User friendly may not be much of a technical issue but helps in better understanding, usage and implementation of cloud services between the user and IoT. A user may not desire to use a particular service if the cloud is not user friendly no matter how better technology be used. IFTTT is another parameter considered for IoT through it may not provide a direct benefits but helps in the integration of additional services available over the internet. If a user is not granted enough storage space and time he may be in high risk of losing his precious data. Hence one must provide adequate storage space and time for user to retain and access particular data. Data computation is another essential factor that draws many user towards IoT, since these devices are generally have less computational power and uploading these primary data to the cloud and utilizing its computational power would be a great deal and the user would save a lot economically. A user will consider IoT only if he is provided necessary security by safeguarding his data and make sure there are no $3^{\text {rd }}$ party interference to his cloud or services. Hence all these factors brings vital conclusions for IoT and hence this paper brings out all the necessary details an IoT user desires.

\section{PROCEDURES AND LITERATURE SURVEY}

\subsection{Calculating Cloud Latency}

Cloud Latency is determined by the time required for the data to reflect on the cloud from the hardware equipment or vice versa [3]. Some clouds control the channels by interrupt driven or by acknowledging when a data variation is observed in the fields. The latency is measured by receiving time stamps from the cloud every second. The time stamp is spawned and circulated by the cloud every second and hence the cloud latency is calculated by summing up the one way travel time of the first packet and the delay taken by every packet with respect to the arrival time of the first packet. The travel time of first packet is calculated by taking half the ping value since ping value is twice the one way travel time. A clock is called when a time stamp is received and this value is subtracted from the clock value of the first received time stamp which results in computation latency. Some clouds do not work in acknowledgement driver process but demands the hardware to desire for the explicit data from the cloud and the requested message is shared with the device. A timer is called to record the local time during request is made and when the data is received. The cloud latency is obtained by the difference in the two clock time obtained from the timer.

\section{Process 1:}

Latency $=($ Ping time $/ 2)+$

(Time taken to receive the ' $\mathrm{N}^{\text {th }}$, time stamp)

- [Time taken to receive 1 st time stamp $+\mathrm{N}$ seconds]

$\Rightarrow$ (Where $\mathrm{N}$ is the number of time stamps received)

\section{Process 2:}

Latency $=($ Time when the requested data message was received) - (Time when the data request was sent)

\subsection{Calculating Throughput}

Packets drop is found by observing an anomaly in the data flow since the data transmitted is correlated by an arithmetic relationship if the cloud libraries are not able to recognize the packet drop in the hardware device [6]. The packet loss is then transformed to throughput percentage and thus illustrated in the standard format.

Throughput $($ in $\%)=$ Packets Lost*100 / Total Packets

\subsection{Interval for subsequent update or Update delay}

Interval for subsequent update or the update delay is the time required by the cloud to receive the forthcoming field value from the hardware equipment with no destruction or loss. The hardware equipment is programmed to forward data values, incremented by a factor of unity and also delay stepping up at the pace of 50 millisecond by the IoT system. The cloud appears to display stable values with no discontinuity after a certain value. That value is accounted to be the reference key to uncover the explicit time delay where the cloud can accept the data. Though some clouds have fixed predetermined values, this paper gives precise update value.

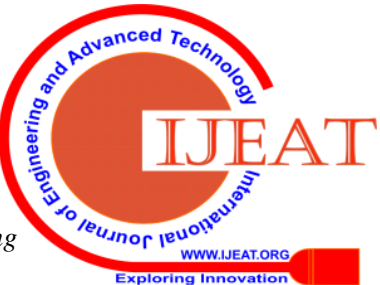




\subsection{User Friendly}

User friendliness of cloud is non quantitative crucial part determined by the cloud's practical use, ease of access to resources, visualization of messages and to extend an online support to the client [7]. The cloud is scored based on the user's convenience to administer widgets, visualizing data, flexibility approach to adapt the clouds resources, feed types, dashboard approved by the cloud server and flexibility to design widgets by the user are essential aspects explored to estimate the value.

\subsection{IFTTT compatibility}

IFTTT is a MQTT broker necessary for its seamless interoperability strength to reach out numerous technologies of diverse platforms from A to Z [8]. Most cloud servers present circuitous mechanism to employ IFTTT using Webhooks which yields a simple URL request of minimal operation. Only a handful of cloud servers offers a direct IFTTT interface.

\subsection{Storage limits}

One of the most remarkable elements demanded by the IoT device is the storage space [5] and time [9] afforded by the clouds for its user since the data is only saved for a precise limit which leads the user to download the data before erasing and hence it is analyzed.

\subsection{Data Computation by clouds}

Data Computation in other hand includes both arithmetical and non-arithmetical steps followed by a well-defined algorithm to get intelligent information [10]. The cloud ought to have features that supports data computation.

\subsection{Service Types}

Majority of the cloud brings out numerous versions of their own cloud with additional features \& benefits based on the users' credits [11]. The cloud favors the user with his credits by upgrading the user's resources to higher levels which can be earned through a financial support and/or by promoting the cloud to other users which calls for a survey on the service types offered by the cloud.

\subsection{Servers}

A cloud system may not operate as a stand-alone server [4], but, to enhance the cloud reliability, redundancy, performance, uninterrupted service, withdrawal for maintenance, client handling capabilities and geographical reach out can extend the cloud with multiple servers design. The server count is resolved by exploring its IP addresses through DNS protocols.

\subsection{Calculating Security Factors}

Protecting critical data and personnel information is something crucial expected by the IoT users [15]. Network security ought to be prioritized in a system configuration because of increasing threats of cyber attackers seeking to breach as many IoT devices as possible. Hence security is

essential in restricting hacker's subversion and intelligence. In this paper security is calculated based on three parameters known as Protocol support [12], Key exchange support [13], Cipher support [14] by employing online tools.

\section{A) Protocol Support}

To analyze protocol support ( $30 \%$ weightage), the cloud's protocol are modelled and the cloud can employ any of the listed protocols (Table 1).

Table I: Protocol Rating
\begin{tabular}{|c|c|c|}
\hline Protocols & $\begin{array}{c}\text { Preferenc } \\
\text { e }\end{array}$ & $\begin{array}{c}\text { Ratin } \\
\mathbf{g}\end{array}$ \\
\hline TLS 1.3 & 1 & 10.0 \\
\hline TLS 1.2 & 2 & 9.5 \\
\hline TLS 1.1 & 3 & 9.0 \\
\hline TLS 1.0 & 4 & 8.0 \\
\hline SSL 3.0 & 5 & 6.0 \\
\hline SSL 2.0 & 6 & 4.0 \\
\hline
\end{tabular}

\section{B) Key Exchange}

An authentication without key exchange permits attacks (MITM, etc.) by hackers that benefits him by gaining access to communication channel and hence the key should be exchanged in a secured way. The key exchange (30\% weightage) considers two operations.

i) One operation is to perform user's verification; the cloud validates the identity of its subscriber and user.

ii) The next step is to assure a secure spawning and transfer /exchange of code words used in the actual transfer of data.

Table II: Key Exchange Ratings

\begin{tabular}{|ll|c|c|}
\hline \multicolumn{2}{|c|}{ Key exchange parameters } & Preference & Rating \\
\hline Key strength (in bits) >= 4096 Ex. 4096 bits & 1 & 10.0 \\
\hline Key strength (in bits) < 4096 & Ex. 3072 bits & 2 & 9.5 \\
\hline Key strength (in bits) < 3072 & Ex. 2048 bits & 3 & 9.0 \\
\hline Key strength (in bits) < 2048 & Ex. 1024 bits & 4 & 8.0 \\
\hline Key strength (in bits) < 1024 Ex. 512 bits & 5 & 6.0 \\
\hline Key strength (in bits) < 512 & Ex. 256 bits & 6 & 4.0 \\
\hline Key strength (in bits) < 256 & Ex. 128 bits & 7 & 2.0 \\
\hline
\end{tabular}

\section{C) Cipher Strength}

A hacker tries to break down transmission by targeting the symmetric cipher first before communication channel. The cloud offers several ciphers ( $40 \%$ weightage) of different types, but higher the bits used for encryption, the more secure is the data transmission.

Table III: Cipher Ratings

\begin{tabular}{|c|c|c|}
\hline Cipher type & Preference & Rating \\
\hline$>=512$ bits Ex. 512 bits & 1 & 10.0 \\
\hline$<=512$ bits Ex. 256 bits & 2 & 9.0 \\
\hline$<=256$ bits Ex. 128 bits & 3 & 8.0 \\
\hline$<=128$ bits Ex. 56 bits & 4 & 4.0 \\
\hline 0 bits (No encryption) & 5 & 0.0 \\
\hline
\end{tabular}

\section{CASE STUDY}

\section{A) Weather Station and Control}

A Weather Station was built to observe and manage indoor environmental parameters. 
A survey was done to achieve IoT in this mini Weather station and was composed of a special sensor board BOOSTXL-SENSEHUB employed with many sensors implanted in a micro-controller TIVA C Series TM4C123GH6PM Launchpad. The sensor board is capable to sense Temperature, Humidity, Light Intensity, Pressure, Precipitation and 3-axis magnetic fields for a good Weather Station. The system was then offered an IoT interface using NodeMCU. The device is also employed to control cooling equipment such as Air Conditioner, humidity through Air Moisturizer and lighting features though Lamps.

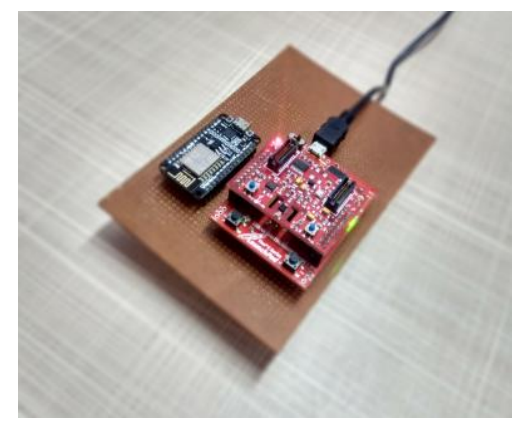

Fig. 1. A. Weather Monitoring System

The clouds were apt to perform logical tasks to control aspects like temperature by turning ON/OFF the Air Conditioner. The system would have functioned with its potential, but few packets were lost and the cloud became less responsive to perform the given task. When the user sought to adjust the device manually through cloud dashboard, some servers were user friendly but some weren't. If the user wants to get notifications when there takes place an unexpected event like humidity is very high through telegram/ web/ twitter/ messaging. The device communicates though these social media platforms which is feasible only through building own widgets or through collaboration between them or through IFTTT. There are numerous aspects to be considered for reliability but one of the aspect is to figure out the number of servers used by the cloud since information like user capacity limits are held classified and is challenging to study on. If the cloud performs all the tasks presented and functions flawlessly, what about the privacy and security of the entire system and the equipment. There arise trust issues that how user may rely on the cloud to guarantee his privacy.

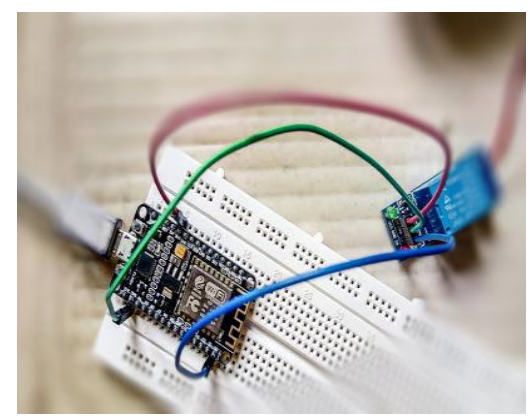

Fig. 1. B. Weather Control System

\section{B) Chemical Tracer}

A chemical tracer was promoted to track chemicals present in the water bodies. The system is cable of handling sensors like $\mathrm{pH}$, Turbidity, Salinity, Dissolved Oxygen, Temperature,
Chemical Ions and Water Depth. The cloud's function is to monitor the sensor values and determine the chemical type present in the water body by computing the data through a well-defined algorithm programmed in the cloud. If the chemical quantity present in the water exceeds a certain threshold limit, the system immediately notifies the abnormality. If suppose the water level of river increases sharply it could mean for a possible flood and a warning message is sent. All these sensors are fixed in a carafe through a probe connecting the functional micro-controller MSP EXP430G2 Launchpad to the IoT device Node MCU through independent codes composed of API's served by the clouds for industrial automation.

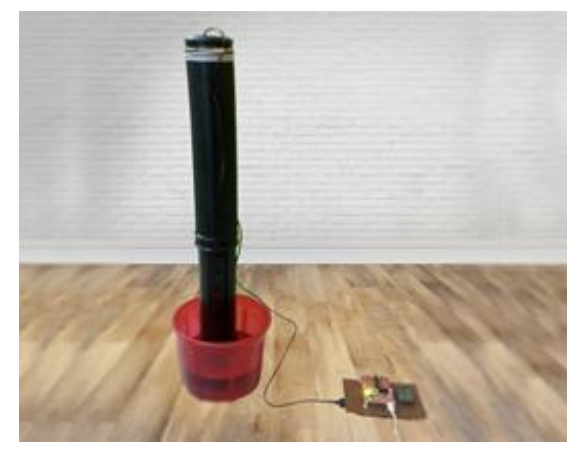

Fig. 2. A. Chemical Tracing System

The chemical tracer was apt to carry out its tasks well as instructed by the program, but there were some troubles encountered in these clouds. Thingspeak, Ubidots and AWS provided room for data computation, performed calculation and detected the type of chemical instantaneously with the collected values. Diverse schemes were employed to upload sensor values at discrete timings and hence the response time reflected by the system was highly unpredictable when moved from one cloud to another as Blynk responded in instantly whereas AWS drew a long time. Another significant aspect was to download the data before crossing the storage limit, but then some clouds save data for days, some months and some years. It is vital to examine the clouds in depth and hence this research is presented to measure deep disrupting factors that cause changes to stability, sustainability, resources and variability of the clouds. The user can analyze the cloud's guidelines presented in this paper and can take up optimal cloud suited for his requirements.

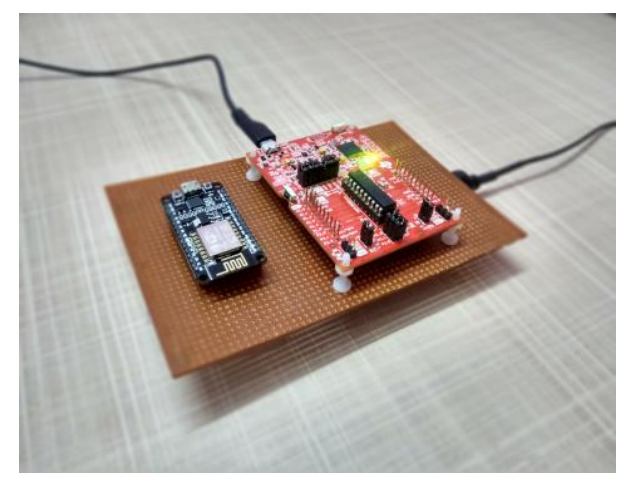

Fig. 2. B. Circuit Diagram 


\section{OBSERVATIONS}

Table IV: Cloud Comparisons

\begin{tabular}{|c|c|c|c|c|c|}
\hline Clouds & Adafruit IO & AWS & Blynk & Thingspeak & Ubidots \\
\hline Total Samples & 336 & 433 & 687 & 577 & 596 \\
\hline Average Latency & $178.67 \mathrm{~ms}$ & $25619.03 \mathrm{~ms}$ & $76.34 \mathrm{~ms}$ & $1255.48 \mathrm{~ms}$ & $1265.07 \mathrm{~ms}$ \\
\hline Maximum Latency & $920 \mathrm{~ms}$ & $35977 \mathrm{~ms}$ & $822 \mathrm{~ms}$ & $2702 \mathrm{~ms}$ & $2891 \mathrm{~ms}$ \\
\hline Minimum Latency & $59 \mathrm{~ms}$ & $10160 \mathrm{~ms}$ & $56 \mathrm{~ms}$ & $849 \mathrm{~ms}$ & $819 \mathrm{~ms}$ \\
\hline Latency Variation & $47 \%$ & $8.2 \%$ & $62.4 \%$ & $26.4 \%$ & $24.2 \%$ \\
\hline Packet Loss & 12 per 1000 & No loss & No loss & No loss & 2 per 1000 \\
\hline Throughput & $98.81 \%$ & $100 \%$ & $100 \%$ & $100 \%$ & $99.83 \%$ \\
\hline Interval for update & $2 \mathrm{sec} \quad(30 \mathrm{data} / \mathrm{min})$ & (any feed) & (any feed) & (32 feeds) & (any feed) \\
\hline Min. update time & $0 \mathrm{sec}$ & $0 \mathrm{sec}$ & $0 \mathrm{sec}$ & $10 \mathrm{sec}$ & $0 \mathrm{sec}$ \\
\hline User Friendly & YES & YES & YES & ACCEPTABLE & YES \\
\hline Widget creation & No creation & Allows creation & No creation & Allows creation & Allows creation \\
\hline IFTTT compatibility & Direct Involvement & Restricts IFTTT & Through Webhooks & Through Webhooks & Through Webhooks \\
\hline Security Rating & $9.65 / 10$ & $9.15 / 10$ & $9.45 / 10$ & $9.45 / 10$ & $9.45 / 10$ \\
\hline Protocol & TLS 1.2 & TLS 1.2 & TLS 1.2 & TLS 1.2 & TLS 1.2 \\
\hline Key Exchange & RSA 3072 & RSA 2048 & RSA 3072 & RSA 3072 & RSA 3072 \\
\hline Cipher Bits & SHA 384 & SHA 256 & SHA 256 & SHA256 & SHA256 \\
\hline Data Computation & None Available & Multiple Platform & None Available & MATLAB & Own Platform \\
\hline Storage Space & $1.5 \mathrm{~GB}$ & $5 \mathrm{~GB}$ & Unlimited & 3 million data / year & Unlimited \\
\hline Storage Time & 1 month & No limits & 3 months & No limits & 2 years \\
\hline Servers & 2 servers & 1 server & 1 server & 2 server & 2 servers \\
\hline IP Address Server 1 & 52.72 .201 .158 & 99.84 .226 .218 & 185.203 .72 .17 & 54.172 .155 .251 & IPV4: 0.23 .124 .67 \\
\hline IP Address Server 2 & 2.7 .124 .212 & & & 54.210 .243 .251 & $\begin{array}{l}\text { IPV6: 2607:f0d0: } \\
\text { 2101:94:0:0:0:4 }\end{array}$ \\
\hline
\end{tabular}

\section{INFERENCE}

The latency of Adafruit IO and Blynk is determined by Equation 1 since the cloud transfers data only if there is any development in the data values. In AWS, Thingspeak and Ubidots, the latency is determined by Equation 2 since cloud opts to transfers the data on request. Adafruit IO shows a small latency considered to be exemplary, but ranges widely since its standard deviation is $47 \%$ of its average latency. The latency of AWS brings down the performance and ranges narrow by its standard deviation of $8.2 \%$. Blynk shows exemplary latency and ranges widely since the standard deviation is $62.4 \%$. The latency displayed by Thingspeak falls into satisfactory levels and its standard deviation is $26.4 \%$ of the average latency. Again, Ubidots shows a satisfactory performance and the latency variation is only $24.2 \%$ of the average latency. Adafruit IO features a packet drop of 12 per 1000 which brings down its performance to nominal levels. Ubidots drops to satisfactory levels since an average of 2 packets are lost in 1000 packets. AWS, Blynk and Thingspeak offer $100 \%$ throughput driving its performance to good levels. Adafruit IO restricts IoT devices to update only at a pace of 30 updates per minute for all the feeds put together. The lowest update time is maintained to be at $0 \mathrm{sec}$ which indicates that the user is granted to update all the 30 values in the early few seconds. Thingspeak restricts IoT devices to update only at intervals of $10 \mathrm{sec}$ for 8 fields (32 channels) which is impressive. Although the officially declared value is $15 \mathrm{sec}$, the users are allowed to update at intervals $10 \mathrm{sec}$ without incurring a packet loss. The users are not permitted to amend the channels unconventionally, but only at periodic intervals, unlike Adafruit IO. AWS, Blynk and Ubidots doesn't restricts IoT devices and the lowest update time is maintained at Osec indicating the user can update values at unconventional time.
Adafruit IO, AWS, Blynk, Ubidots provides user-friendly elements like visualization of inputs through multiple widgets, public sharing, community sharing, activity tracking, key regeneration, carrying out triggers, dashboards, histogram plot, managing feeds for both upload and download. Adafruit IO shows distinct features such as Weather update, maps and remote control, but there are no features to create new widgets by the user. AWS allows multiple platforms of its own but doesn't allows any other applications that are not of Amazon service which shows the dominating nature of the cloud. Blynk provides awesome widgets and gadgets such as joystick, video streaming, email, music player, GPS stream, etc. Blynk offers service only through its App which works both in Android as well as in IOS, but doesn't offer any website services. Ubidots has awesome aspects such as html canvas, video streaming, maps, pie chart, email, clock widget, joystick, etc. and also provides both website and apps which improvises user friendliness. Thingspeak has limited widgets, but provides platform for the user to design his own widgets. Adafruit IO affords a leading IFTTT service which is an exceptional feature contrast to other clouds by providing editable options in IFTTT website itself. AWS do not offer IFTTT service, neither supports an indirect interface through Webhooks but is supported by Amazon Alexa and AWS Lambda. AWS build Alexa and Lambda in its own platform to perform operations like voice controlling, home automation which shows dominating features of the cloud. Blynk, Thingspeak and Ubidots affords IFTTT service by an indirect interface through Webhooks which limits the clouds features.

The data stored in all the cloud (Adafruit IO, AWS, Blynk, Thingspeak and Ubidots) are constrained. If the data exceeds the allocated storage limit, the cloud overwrites on the prevailing data which prompts the user to back up the data periodically or extend storage by paid subscription.

Adafruit IO offers an extension up to 2 month storage from a 
monthly storage. AWS brings an extension up to $10 \mathrm{~GB}$, $15 \mathrm{~GB}$ or the user is billed for every extra GB. Ubidots offers an excellent period for the users to download the data and hence provides the best storage options. Blynk provides unlimited storage space with 3 months storage time. Through unlimited time is offered, feeds are bought for virtual currency which Blynk calls it Energy. Blynk stores each data on every feed that costs fixed energy based on the widget's complexity and functions. Hence limited feed provide limited storage. Thingspeak provides limited storage facilities of 3 million messages per year for all the channel with no duration limit. A paid subscription brings out numerous additional features. Adafruit IO and Blynk provides basic logical computation and triggers. The cloud demands the user to download the data and perform his own algorithm or computation offline. Hence Adafruit IO and Blynk is not appropriate for operations where user desires an online computation. This feature tends to bring down the capabilities of these clouds. AWS provides excellent logical, arithmetic platform for computation and is capable of computing complex algorithms and arithmetic operations and results are given back for the systems routine functions. Thingspeak also provides excellent logical, arithmetic platform for computation using MATLAB, and time \& triggers events by the user's choice. Ubidots provides satisfactory logical, arithmetic platform for computation using its own platform. The cloud allows IoT system to compute simple algorithms and arithmetic operations and results are given back for its routine functions.

Adafruit IO benefits users through a paid subscription which would break the clouds restrictions of using 10 channels and 5 dashboard to unlimited channels and dashboards. The data upload speed is also increased from 30 data points to 60 data points and the storage is extended to 2 months. AWS uses various applications like real time clock services, Amazon EC2 and Amazon RDS through paid subscription. Storage can also be extended through payment that brings an extension unto $10 \mathrm{~GB}, 15 \mathrm{~GB}$. Amazon provides a second way by charging for every additional GB consumed by the user. Blynk uses its own virtual currency called energy. Blynk provides 2000 energy at free of cost. Each widgets and feeds costs a fixed energy based on its complexity \& functions and hence the user can purchase suitable widgets for energy. When the user doesn't use a particular widget and wishes to delete it, Blynk returns the energy. Using this strategy Blynk charges energy with nominal fare once forever unlike many clouds. Thingspeak offers various benefits through one-time subscription license that costs $\$ 650$ which extends storage from 3 to 33 million messages/year, $10 \mathrm{sec}$ to $1 \mathrm{sec}$ update time, 4 to 250 channels, $20 \mathrm{sec}$ to $60 \mathrm{sec}$ computational time, 3 to 50 MQTT subscriptions, 3 to unlimited private sharing and an additional support provided by Standard Math Works Support. Ubidots provides no separate versions of its cloud by restricting its resources, but provides 1 month free trial to taste its extensive elements and later proceed through a paid subscription. Ubidots also provides paid services such as Reports, Ubifunctions and White Labels that are used for computation purposes. Subscription plan varies by the no. of feeds connected to the cloud and a minimum plan of 10 feeds costs $\$ 20$ and a good plan of 5000 feeds costs about $\$ 2499$ a month. If the trial period is over, the feeds are blocked.
Adafruit IO, Thingspeak offers dual servers that works on IPV4 basis. While AWS and Blynk offers only one IPV4 server. Ubidots offers dual servers. One of the servers operates on IPV4 basis and another on IPV6 basis. I wonder why the clouds have employed TLS 1.2 and haven't yet updated their servers to TLS 1.3 protocol which seems to be a better protocol. Adafruit IO utilizes secured protocols that puts the analyzed value to be $9.65 / 10$ which shows up the clouds security elements are excellent. Using SHA 384 cipher bits and RSA 3072 key exchange boosts the security parameter. We anticipate the security parameter of AWS to be 9.15 which shows up the clouds security elements are satisfactory. The cloud displays nominal security protocols like RSA 2048, but still AWS compensates the security using multiple keys making it a better firewall for security breech. The security parameter of Blynk, Thingspeak and Ubidots is estimated to be 9.45 which shows up the clouds security elements are good. All clouds employs RSA 3071 which seems to be a better key exchange protocol than AWS that uses RSA 2048.

\section{RESULTS AND DISCUSSION}

\section{A) Adafruit IO}

Adafruit IO serves best for microscopic IoT applications in Industrial and Home Automation which desires for minimal resources and channels suited for time and trigger based applications. Adafruit IO is a user friendly cloud with diverse resources and accessibility, possesses less latency, minimum update time, outstanding security and usage of dual servers. The cloud provides user friendly aspects such as the visualization of data through multiple widgets, public sharing, community sharing, activity tracking, trigger events and to support distinct feeds, SMS and E-mail posting tasks.

Pros:

Adafruit IO is exceptionally good in promoting full-fledged graphic visualization tools to apprehend the device's activities on the screen. The cloud has good latency and minimum update time. Another remarkable aspect contrast to other clouds is that the server offers a leading IFTTT service that enhances the accessibility of cloud. The cloud shows exceptional characteristics by employing secured protocols enhancing privacy and security. The cloud provides dual servers which improvises the reliability.

Cons:

Adafruit IO provides an average data update speed, lacks usage of synthetic variables and leaves no room for data computation. The cloud offers limited data feeds, dashboard and resources and hence the clouds' applications are confined to small scale automation. The cloud's performance is troubled by the throughput ratio since the packet loss is immense. Although the latency of the cloud is good, the latency variation is unpredictable and hence unstable. The cloud restricts the user to have a new widget by his choice. The storage space and time is inadequate for an IoT device that demands high storage for its complex function.

\section{B) $A W S$}

AWS is a combination of crucial frameworks that hooks up any equipment from sensors to gateways and yields business value to IoT. The security is enhanced by using multiple call

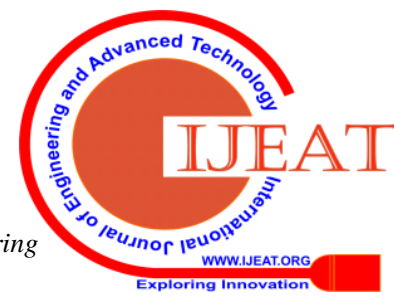


and subscriber functions. AWS uses multiple keys such as AWS key ID, AWS secret key, AWS IoT region, endpoint and domain that contributes an end-to-end security for all devices, data, and heterogeneous connectivity. The server has the ability to handle incoming real-time data streams with event filtering, correlation and aggregation that serves perfect for large-scale Industrial and Home Automation.

\section{Pros:}

AWS is exceptionally good in Data computation that favors the user to perform a complete evaluation using an array of intrinsic platforms. AWS doesn't restrict devices connected to the cloud and supports multiple IoT devices to have an effective transfer of data. The cloud's latency variation is at minimal levels. The cloud yields excellent throughput which implies zero packet loss and flags transfer of critical information through AWS. Alexa supported in electronic equipment facilitates voice based applications.

\section{Cons:}

The latency of AWS is extremely high which narrows down its operations. AWS shows dominating instincts that doesn't support external applications to change its purpose or operations. The clouds security parameters are disturbed by using average protocols and encryptions. AWS doesn't support IFTTT interaction which limits external applications that are not in AWS's boundary.

\section{C) Blynk}

Blynk is a popular mobile data visualization cloud. Blynk captures data through its servers and offers to stream through mobile platform and acts on massive amounts of incoming data series and plots instantly when it reaches the cloud servers. Blynk offers flexible sample time based on the user's requirements, customizable drag and drop facility is enhanced when live charts and grids are embedded with user needed forms. Event Monitoring, location tracking aid mobile application are easily maintained with customizable Email and SMS that suits best for large scale non-computational Industrial and Home Automation.

\section{Pros:}

Blynk is filled with diverse widgets that goes with most real-time applications, excellent visualization of received data, multiple widgets, low latency and app based functioning, nominal one time fare for feeds, outstanding average latency, update time and throughput. The storage time provided by the cloud is likewise attractive since three month gives a user the credibility to have ample time to pull data from their account. Blynk aids with its exclusive widgets such as the music player, twitter, joystick, Accelerometer, GPS trigger, SMS notification, Email trigger, etc.

\section{Cons:}

Blynk has no room for data computation, lacks usage of synthetic variables and is also a solo server app based cloud which is not supported by browsers. The cloud shows unstable variation in latency and doesn't allow to create widgets of user's choice and IFTTT is indirectly supported through Webhooks.

\section{D) Thingspeak}

Thingspeak is an IoT cloud platform dedicated for technology and clients. ThingSpeak permits real-time data compilation, evaluation and actuation with write and read API's. Embedded with channel monitoring data storage, plugins, visualization and integration of the client's data with a range of external platforms, comprising Talkback, Plugin, Arduino, ThingTweet, ThingHTTP and MATLAB. Thingspeak bears all kinds of data, location, and status that suits best for non-controlling Industrial and Home automation.

\section{Pros:}

Thingspeak affords a noble latency with a slight fluctuation. The cloud throughput ratio is excellent and gratifies the user to transmit significant data to the equipment and cloud. Thingspeak allows the user to establish widgets by user's choice and presents features like Time control app (performs scheduled operations), React app (performs event based services) and Tweet control (replies to the Twitter messages). The cloud allows real-time data computation using MATLAB and follows an ethical system of not eliminating the user's data. Thingspeak offers dual server to improve its reliability. Cons:

Thingspeak displays less user friendly elements in contrast with other clouds. Although the cloud supports designing widgets, there are only a handful of built-in widgets with minimal functions and visualization. Thingspeak emphasizes a large update time for a specific feed and also expects the user to physically enter the updated feed values in a browser. The cloud means a circuitous IFTTT interaction through Webhooks and grants a minimal memory which doesn't suit for large-scale applications unless upgraded.

\section{E) Ubidots}

Ubidots is associated for devices that implements discrete applications, explores case studies, surveys field implications, and shares data. The cloud provides excellent connectivity, control, monitoring, and operations between IoT devices and resources. Its mission is to legitimize communities to pat IoT for expanding its revenue streams through enhanced client satisfaction and increase potential data from remote devices and suits best for industrial automation.

Pros:

Ubidots displays user friendly elements, adopts synthetic variable and performs data computation. The cloud shows a decent latency that exhibits fewer variations. Ubidots has minimum update time provides amenities for the user to model his own widgets and employs protocols that features good security and preserves the user's privacy. Ubidots employs its own platform for data computation and employs synthetic variables. Ubidots offer unlimited data storage which is a unique aspect contrasting other clouds and affords substantial storage time for its users. The cloud employs dual server of IPV4 and IPV6 servers for the user's comfort.

Cons:

Throughput percentage of the cloud brings down the effectiveness of cloud. The cloud supports indirect usage of IFTTT interface though Webhooks and demands high financial costs for regular operation which limits its service and users.

\section{CONCLUSION}

IoT in Industrial and Home Automation is turning up to meet the present day needs efficiently with an enormous capabilities and benefits. We analyzed five of the most eminent clouds Adafruit IO, AWS, Blynk, Thingspeak and Ubidots based to pursue distinct elements of the cloud for a

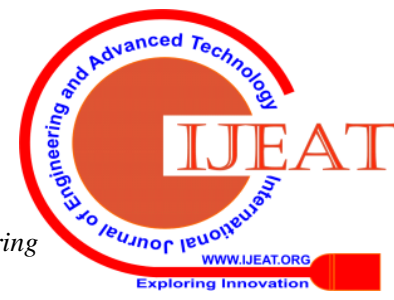


quality IoT interface. Home and Industry Automation needs smooth implementation for an excellent quality of service data transmission for all its operations. Majority of the applications needs functions like controlling, data uploading / monitoring / computation, voice controlling, platform switching, trigger or time based application, email / messaging and sometimes Block chain which demands special resources and flexibility. If the user desires for a unique application that doesn't fall under these categories, the user can analyses the cloud features by following the procedures surveyed in this paper and decide the cloud that suits best for his purpose and needs. Based on the analysis done we are recommending the open source cloud to adapt for the following parameter value for the users to have the best experience in using the cloud platform.

Table V: Recommended Standard

\begin{tabular}{|l|l|}
\hline \multicolumn{1}{|c|}{ Parameters } & \multicolumn{1}{c|}{ Recommended Values } \\
\hline Latency & $250 \mathrm{~ms} \pm 50 \mathrm{~ms}$ \\
\hline Throughput & $100 \%$ \\
\hline Interval for update & $2 \mathrm{sec}(30 \mathrm{data} / \mathrm{min})$ \\
\hline IFTTT compatibility & Direct Involvement \\
\hline Protocol & TLS 1.3 \\
\hline Key Exchange & RSA 3072 \\
\hline Cipher Bits & SHA 384 \\
\hline Data Computation & Average Calculations \\
\hline Storage Time and Space & 4 months; 1.5 GB / month; \\
\hline Servers & 2 servers \\
\hline
\end{tabular}

\section{REFERENCES}

1. Abdur Rahim Biswas, Raffaele Hiaffreda: IoT and Cloud Convergence: Opportunities and challenges in IEEE world forum on internet of things (WF-IoT), pp. 375 - 376, 24 April 2014.

2. Partha Pratimray; A survey of IoT cloud platforms in Future computing and informatics journal, pp. 35-46, December 2016.

3. Luciano Barreto, Antonio Celesti, Massimo Villari, Maria Fazio, Antonio Puliafito in An Authentication model for IoT clouds, IEEE/ACM International conference on Advances in Social networks Analysis and Mining (ASONAM), 11 February 2016.

4. Ran Canetti, Ben Riva, Guy N. Rothblum, Practical Delegation of Computation using Multiple servers, proceedings of the 18th ACM conference on Computer and Communications Security, pp. 445-454, 21 October, 2011.

5. Boyi Xu, Lihong Jiang, Athanasios V, Vasilakos, IoT-Based Big Data Storage Systems in Cloud Computing: Perspectives and Challenges in IEEE Internet of Things Journal, pp. 75 - 87, 20 October 2016.

6. Nirandika Wanigasekara, Jenny Schmalfuss Darren Carlson, David S. Rosenblum, A bandit approach for intelligent IoT service composition across Heterogeneous smart spaces in proceedings of the 6th International conference on IoT, pp. 121-129, 7 November 2016.

7. Yuang Chen, Thomas Kunz; performance evaluation of IoT Protocols under a Constrained Wireless Access Network in International conference on selected topics in Mobile \& Wireless networking (MOWNET), pp. 1 - 7, 23 June 2016.

8. Davide Mulfari, Antonio Celesti, Massimo Villari, A Computer System Architecture providing a User-Friendly Man machine interface for accessing Assistive Technology in Cloud computing in Journal of Systems and Software, pp. 129-138, February 2015.

9. Steven Ovadia, Automate the Internet with "If This Then That" (IFTTT) in Behavioral \& Social Sciences Librarian, vol. 33, 2014, pp. 208-211, 10 November 2014.

10. Qi Zhang, Lu Cheng, Raouf Boutaba, Cloud computing: state-of-the-art and research challenges, in Journal of Internet Services and Applications, pp. 7-18, 20 April 2010.

11. Zia ur Rehman ; Farookh K. Hussain ; Omar K. Hussain, Towards Multi-criteria Cloud Service Selection in 2011 Fifth International Conference on Innovative Mobile and Internet Services, Ubiquitous Computing, pp. $44-48,4$ August 2011.
12. Savio Sciancalepore, Angelo Capossele, GiusepPe Piro, Gennaro Boggia, Giuseppe Bianchi, KEY MANAGEMENT PROTOCOL WITH IMPLICIT CERTIFICATES FOR IOT SYSTEMS IN IOT-SYS '15 PROCEEDINGS OF THE 2015 WORKSHOP ON IOT CHALLENGES IN MOBILE AND INDUSTRIAL SYSTEMS, PP. 37-42, MAY 18, 2015;

13. Yosra Ben Saied, Alexis Olivereau; HIP Tiny Exchange (TEX): A distributed key exchange scheme for HIP-based Internet of Things in Third International Conference on Communications and Networking, pp. 1 - 8, April 1, 2012

14. Mouza Bani Shemaili, Chan Yeob Yeun, Khalid Mubarak, Mohamed Jamal Zemerly, A new lightweight hybrid cryptographic algorithm for the IoT 2012, International Conference for Internet Technology \& Secured Transactions, pp. 87 - 92, 12 December 2012.

15. Sachin Babar, Parikshit Mahalle, Antonietta Stango, Neeli Prasad, Ramjee Prasad, Recent Trends in Network Security and Applications in International Conference on Network Security and Applications, CNSA 2010, pp. 420-429, 17 November 2016.

16. Urs Hunkeler, Hong Linh Truong, Andy Stanford-Clark, MQTT-S - A publish/subscribe protocol for Wireless Sensor Networks in 3rd International Conference on Communication Systems Software \& Middleware and Workshops, pp. 791-798, 27 June 2008.

17. Jayavardhana Gubbi, RajkumarBuyya, SlavenMarusic, MarimuthuPalaniswami, Internet of Things (IoT): A vision, architectural elements, and future directions in Future Generation Computer Systems, pp. 1645-1660, September 2013.

18. Jalamkar, D., Krishnakumar, A. A. Selvakumar. "Implementation of Internet of things in a mobile Humanoid robot: A base for future of IOT enabled robotics applications" in International Journal of Engineering \& Technology 7, vol. 4, pp. 386-389, 2018.

19. Jalamkar, D., and A. A. Selvakumar. "Use of internet of things in a humanoid robot-a review." in Advances in Robotics \& Automation 5, vol. 2, pp 2-5, 2016.

20. Antonio Celesti, Maria Fazio, Maurizio Giacobbe, Antonio Puliafito, Massimo Villari; Characterizing Cloud Federation in IoT; $30^{\text {th }}$ International Conference on Advanced Information Networking \& Applications Workshops (WAINA), pp. 93-98, 19 May 2016.

21. G.M. Lee, The Internet of Things - Concept and Problem Statement in Institute TELECOM, pp. 21, 2012.

\section{AUTHORS PROFILE}

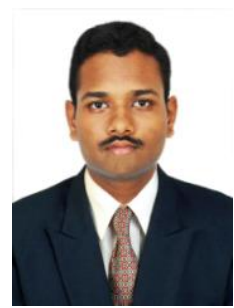

Aravindh Kolanchinathan, a student pursuing his $4^{\text {th }}$ year, Bachelor of Technology ECE with honors in Vellore Institute of Technology, Chennai, India. He has keen interest in Embedded System, Internet of Things, Image Processing, Cloud services, Applications of Machine Learning and Full Stack Development. He was Co-Leader, Semi-finalist and Technical Head for the Start-up funded by DST, Govt. of India and Texas Instruments in IICDC 2018. Has keen interest in technologies like Quadcopter, IoT, LIC, Neural Networks, Robotics and Automation, etc.

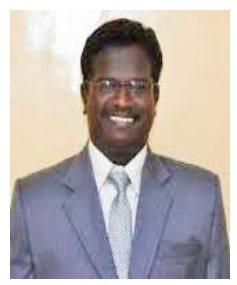

Dr. Arockia Selvakumar A, is member of Design and Automation Research Group at the Vellore Institute of Technology, Chennai where he is working as a Senior Associate Professor of School of Mechanical and Building Sciences. He holds a lifetime membership of RSI and a member of IEEE. His field of interest includes Robotics and Automation, IOT, Bio-Mechanics and Finite Element analysis. 